\title{
Mobility-dependent small-scale propagation model for applied simulation studies
}

\author{
Dmitri Moltchanov
}

\begin{abstract}
We propose an extension to existing wireless channel modeling techniques introducing a notion of mobility behavior of the user. We represent large-scale propagation characteristics of wireless channels as a mobilitydependent stochastic process that explicitly tracks the movement of the user between areas with different received local average signal strength (RLASS). Our model consists of two different parts: mobility model and propagation model. Mobility of the user is modeled by a Markov chain with finite state space. Large-scale propagation characteristics of wireless channel are represented as a function of mobility model. Small-scale propagation characteristics are then obtained taking into account shadowing of the line-of-sight propagation path. Based on the amount of available information regarding a given landscape environment we develop two different parametrization methods (i) RLASS values are available for limited number of points in a given landscape and (ii) RLASS information is not available. The model is suitable for simulation studies of applications' performance in presence of RLASS changes caused by movement of the user.
\end{abstract}

\section{Introduction}

To estimate performance of wireless channels propagation models are often used. We distinguish between large-scale and small-scale models. The former models capture propagation characteristics on a coarse granularity using the notion of the received local average signal strength (RLASS), see e.g., [1-3]. Models characterizing rapid changes of the received signal strength are called small-scale propagation models, see e.g., [4-6]. These models capture propagation characteristics on a finer granularity.

Neither large-scale nor small-scale models take into account the signal strength attenuation caused by movements of a user. To be precise small-scale propagation models do take into account the so-called small-scale mobility of the user over short distances [7]. Such models describe rapid fluctuations around a constant mean which are called fading. Such processes are implicitly assumed to be stationary at least in the second-order sense and mean equals to the received local average signal strength (RLASS). However, if we would consider larger travel distances (i.e., more than just few meters) RLASS starts to vary and the most important factors

Correspondence: moltchan@cs.tut.fi

Department of Communication Engineering, Tampere University of Technology, P.O. Box 553, Tampere, Finland affecting it are terrain, speed of a mobile and distance from between transmitter and the receiver. In this article, contrarily to most small-scale propagation models proposed so far, we explicitly take into account two of these three factors-terrain and mobility of a user over it.

In a mobile environment a user is allowed to change its position at any instant of time and these movements are not restricted to short travel distances. A receiver during a single session experiences different propagation characteristics. To predict these changes, an adequate model must capture both movement of a user between areas with different propagation characteristics and small-scale propagation characteristics in each area. Propagation characteristics must be represented as a probabilistic function of user's movements. Such model would be useful to estimate performance provided to applications while user is in traveling mode.

The rest of the article is organized as follows. In Section 2, we describe the structure of the model and introduce our modeling assumptions. Then, in Section 3, we propose three different parametrization methods for our model. Extensions and refinements of the model are discussed in Section 4. Finally, in Section 5 we test some of our assumptions. Conclusions are provided in the last section. 


\section{Structure of the model}

\subsection{Preliminary notes}

The received signal strength in wireless networks is basically affected by three factors: (i) type of the terrain we consider (ii) speed of mobiles, and (iii) distance between the transmitter and receiver. Most small-scale propagation models proposed so far either implicitly or explicitly assumed that all these factors are fixed, i.e., a certain type of terrain is considered, speed of mobiles as well as distance between transmitter and receiver are fixed. The main reasons is that even with these assumptions the resulting received signal strength is a covariance stationary stochastic process with complicated distributional and correlational properties. The reason for such reduced complexity of models is that smallscale propagation models were mainly used to design transceivers and associated lower layer techniques. For such tasks these models provide sufficient accuracy and should not be extended to more complex cases.

When performance of information transmission at higher layers is concerned we are interesting in adding those additional factors to the small-scale propagation models. Indeed, during the active session a mobile user may move inside and in-between cells experiencing not only qualitatively different propagation characteristics (e. g., different distributions but the same mean) but quantitatively different too (e.g., different RLASS). Thus, in addition to the type of small-scale propagation at a certain separation distance from the transmitter we also have to take into account movement of a user between areas with different RLASS. We would like to note that allowing at least one factor affecting the received signal strength to be variable may result in complex stochastic process making the model applicable for simulation studies only.

\subsection{The mobility model}

Assume that a given cell of a circular shape is divided into a finite number of areas, $M$, such that these areas are non-overlapped and the sum of their areas equals to the area of the cell. Each area is associated with a certain, not necessarily distinctive value of RLASS. We assume that a mobile user may probabilistically move between these areas.

Consider a discrete-time environment, i.e., time axis is slotted, the slot duration is constant and given by $\Delta t$. Changes of areas are only allowed at the slot boundaries. To capture movement of a user between areas we use a homogenous discrete-time Markov chain (DTMC) $\left\{S_{P}\right.$ $(n), n=0,1, \ldots\}, S_{P}(n) \in\{1,2, \ldots, M\}$, whose states correspond to areas. Let $D_{P}$ be its transition probability matrix. To parameterize this model $M, D_{P}$, and $\Delta t$ shall be provided.

\subsection{The large-scale propagation model}

RLASS is mainly a function of the distance between the transmitter and the receiver and the type of propagation environment. To represent it, we associate a value of RLASS with each state of the mobility model. Let $\{P(n)$, $n=0,1, \ldots\}, P(n) \in\left\{P_{1}, P_{2}, \ldots, P_{M}\right\}$ be the RLASS process whose underlying Markov chain is $\left\{S_{P}(n), n=\right.$ $0,1, \ldots\}$, i.e., the value of RLASS is modulated by the underlying Markov chain. To parameterize this model we have to provide the RLASS vector $\vec{P}=\left(P_{1}, P_{2}, \ldots, P_{M}\right)$.

\subsection{The small-scale propagation model}

For performance evaluation studies, small-scale propagation characteristics in a certain area can be modeled by doubly-stochastic process $\left\{R_{i}(n), n=0,1, \ldots\right\}$ modulated by homogenous DTMC $\left\{S_{R, i}(n), n=0,1, \ldots\right\}, S_{R}$, ${ }_{i}(n) \in\left\{1,2, \ldots, H_{i}\right\}$, each state of which is associated with conditional probability distribution function of the received signal strength $F_{R, i}(k \Delta f \mid j)(\Delta f)=\operatorname{Pr}\left\{R_{i}(n)=k \Delta f \mid\right.$ $\left.S_{R, i}(n)=j\right\}, k=1,2, \ldots, N, j=1,2, \ldots, H_{i}$, where $N$ is the number of bins to which the signal strength is partitioned, $\Delta f$ is the discretization interval. The slot duration $\Delta t$ of the model equals to the time to transmit a single bit at the wireless channel. Slot durations of mobility and small-scale propagation models are equal and synchronized.

To capture small-scale propagation characteristics we distinguish between LOS and NLOS environments. In the former case the small-scale propagation envelop distribution is Rician. As the dominant component fades away the small-scale propagation envelop distribution degenerates to Rayleigh distribution. Thus, small-scale propagation characteristics in different areas are, at least, qualitatively (distribution) or quantitatively (RLASS) different. Therefore, areas must be determined such that each of them is uniquely characterized by mean/distribution pair. Then, each area must be associated with unique small-scale propagation model $\left\{R_{i}(n), n=0,1, \ldots\right\}, i=1$, $2, \ldots, M$ such that $E\left[R_{i}\right]=P_{i}$, i.e., the RLASS in the area $i$ is the mean of $\left\{R_{i}(n), n=0,1, \ldots\right\}$. We require that the state space of all small-scale propagation models is the same and given by $\{1,2, \ldots, H\}$.

Since propagation characteristics are a probabilistic function of the movement between areas, the choice of the 'active' propagation model $\left\{R_{i}(n), n=0,1, \ldots\right\}, i=$ $1,2, \ldots, M$, in the slot $n$ depends on the area $i$ in the 
slot $n$, i.e., $\{R(n), n=0,1, \ldots\}=\left\{R_{i}(n), n=0,1, \ldots\right\}, S_{P}$ $(n)=i$. Recalling that all small-scale propagation models were assumed to have the same number of states, $H$, the state-space of the resulting model is

$$
S_{R}(n) \in\{(1,1), \ldots,(1, H), \ldots,(M, 1), \ldots,(M, H)\} .
$$

An appropriate small-scale propagation model is only associated with the state of the mobility model corresponding to the appropriate area. Hence, transition probabilities of DTMC of mobility-dependent model is given by

$$
d_{R, i j}=d_{R, i, k j}, k, j \in\{1,2, \ldots, H\}, S_{P}(n)=i .
$$

To parameterize mobility-dependent small-scale propagation model we shall provide $H, D_{R, i}, F_{R, i}(k \Delta f j)(\Delta f)$, $i=1,2, \ldots, M, k=1,2, \ldots, N, j=1,2, \ldots, H$.

\section{Parametrization of the model}

\subsection{The mobility model}

Assume that the number of areas $M$ and associated RLASS vector $\vec{P}$ are known. It is fair to expect $M P$ that the mean sojourn time in a certain area depends on its size. Consider a user that is in the area $i$ in the slot $n$. In the next slot this user may stay in the area $i$ or move to another area. The only areas to which a user can move in a slot are adjacent areas denoted by $\Omega_{i}$. Consider areas from $\Omega_{i}$ as a single area. We compute transition probability between area $i$ and $\Omega_{i}$ as

$$
d_{L, i \Omega_{i}}=\frac{\sum_{\forall i \in \Omega_{i}} S_{j}}{S_{i}+S_{\Omega_{i}}}, \quad d_{L, i i}=\frac{S_{i}}{S_{i}+S_{\Omega_{i}}},
$$

where $S_{i}$ is the area of area $i, S_{\Omega i}$ is the area of $\Omega_{i}$, $d_{L, i \Omega_{i}}, i=1,2, \ldots, M$, are transition probabilities between area $i$ and $\Omega_{i}$. Depending on the length of the border between area $i$ and areas from $\Omega_{i}$ transition probability $d_{L, i \Omega_{i}}$ is distributed among transition probabilities $d_{L, i j}, j \in \Omega_{i}$, as

$$
d_{L, i j}=\frac{d_{i \Omega_{i}} V_{i j} w_{i j}}{\sum_{\forall j \in \Omega_{i}} V_{i j}}, \quad j \in \Omega_{i},
$$

where $V_{i j}, j \in \Omega_{i}$ are lengths of the border between $i$ and $j$. Parameters $w_{i j}, j \in \Omega_{i}$ are introduced to represent directional movement of the user in a certain application scenario, e.g., highway, city center. Since this movement is specific for a given environment, no general expression can be provided. Note that $\sum_{j \in \Omega_{i}} w_{i j}=1, i=1,2, \ldots, M$.

\subsection{Large-scale propagation model}

\subsubsection{Parametrization based on measurements}

Measurements of RLASS are often given by the 3D vector $\vec{P}_{X Y}=\left(x_{i}, y_{i}, P_{i}\right), i=1,2, \ldots, M$, where $M$ is the number of measurements, $\left(x_{i}, y_{i}\right)$ is the coordinate of $i$ th measurement and $P_{i}$ is the RLASS value of respective measurement.

Practically, it is not feasible to measure RLASS in each and every point of the landscape. Thus, information given by $\vec{P}_{X Y}$ is often insufficient to parameterize our model. To estimate $\vec{P}$ and $M$ we have to determine areas to which these measurements belong to, such that the approximation error is minimized. To determine areas we propose to use a division of the cell into areas whose vertexes are measurement points $\left(x_{i}, y_{i}\right), i=1,2$, ..., M. An appropriate division of the cell is given by Voronoi (Dirichlet) tesselation separating a region $E$ of the space $\mathfrak{R}^{2}$ into polygons $E_{i}, i=1,2, \ldots, M$, using $M$ points drawn from a certain point process. In our case the space is $\mathfrak{R}^{2}$ and coordinates of measurements are considered as a realization of the point process. For each measurement point $\left(x_{i}, y_{i}\right), E_{i}$ is the area consisting of all locations that are closer to $\left(x_{i}, y_{i}\right)$ than to any other point. There are a number of algorithms to compute Voronoi tesselation [8].

To determine areas with different RLASS it is not strictly required to distinguish between values of RLASS in each area. Instead, it is possible to consider ranges of RLASS. In this case the range $\left(\max _{\forall i} P_{i}-\min _{\forall i} P_{i}\right)$ must be divided into $K$ non-overlapping ranges of length $\Delta P$ $=\left(\max _{\forall i} P_{i}-\min _{\forall i} P_{i}\right) / K$. Then, all measurements are classified to these ranges. Adjacent areas having RLASS values falling in the same range can be grouped. An example is shown in Figure 1, where measurements points, the Voronoi tesselation, and resulting areas are shown.

\subsubsection{Theoretical parametrization}

Measurements of RLASS are often unavailable. In this case, we can parameterize our model based on approximate estimation of shadowed areas and subsequent application of large-scale propagation models.

Assume that centers of shadowers are distributed according to the stationary Poisson point process with mean $\lambda$. The parameter $\lambda$ depends on a given landscape. Note that whenever possible actual placements of shadowers can be used. We also assume that (1) shadowers are of rectangular form, (2) thickness of shadowers is zero, (3) their widths and heights are arbitrary distributed, (4) height of the transmitter antenna $h_{a}$ is known.

Three possible shadow placements caused by shadowers with different relation between initial parameters are shown in Figure 2, where $d_{s}$ is the shadow length, $R$ is the radius of a cell, $d$ is minimal distance between the center of the cell and a shadower, and $h_{s}$ is the height of shadower. We should distinguish between three cases: (1) $h_{a}>h_{s}, d_{s} \leq R-d$, the shadow is within the cell, (2) $h_{a}>h_{s}, d_{s}>R-d$, the shadow is bounded 


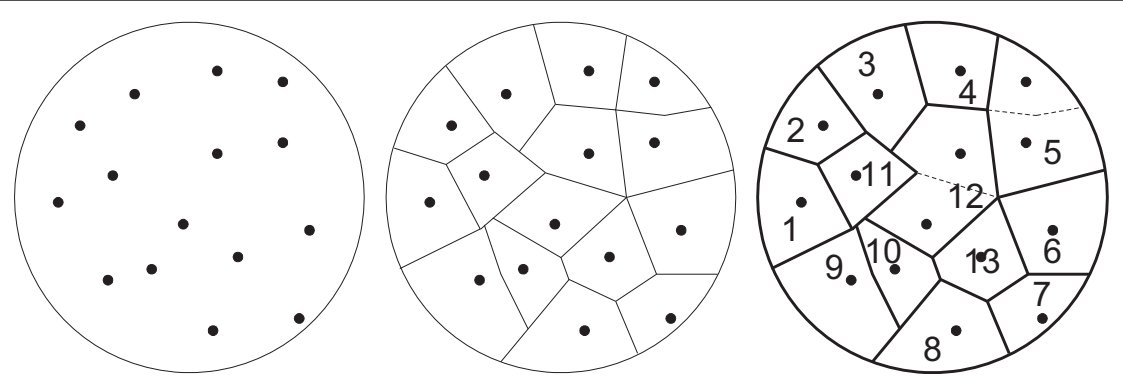

Figure 1 Example of division of a cell based on measurements.

outside, (3) $h_{a}<h_{s}$, the shadow virtually continues up to infinity. Since we consider a single cell, the latter two cases can be treated similarly.

The area of an arbitrary shadow is given by

$$
S_{s}=\min \left(4 \pi R^{2} \frac{\gamma}{360}, 4 \pi\left(d+d_{s}\right)^{2} \frac{\gamma}{360}\right)-\frac{d w_{s}}{2},(5)
$$

where

$d_{s}=\min \left(\frac{h_{s}}{\cot \beta}, R-d\right), \beta=90^{\circ}-\arctan \frac{d}{h_{a}-h_{s}}$.

To estimate RLASS vector $\vec{P}$ we use large-scale propagation models. It was shown in [9] that the mean value of propagation loss, $L(d)$, can be approximated by

$$
E[L(d)]=L_{s}\left(d_{0}\right)+10 n \lg \left(\frac{d}{d_{0}}\right)+X_{\sigma}
$$

where $d$ is the separation distance, $n$ is the path loss exponent, $d_{0}$ is the standard distance, $L_{s}\left(d_{0}\right)$ is the propagation loss at $d_{0}, X_{\sigma}$ is the factor unique for a given environment.

For every shadowed area the propagation loss is estimated using (6) with $n>2$, for unshad- owed areas $n \approx$ 2. Parameters for different frequency bands are given in $[2,10]$. Given a certain transmission power of the base station, propagation loss can be related to RLASS [7].

Observe that there can be shadows whose length is only slightly less than the radius of the cell. The range of RLASS corresponding to these shadows is large leading to modeling errors. To avoid it, in addition to division of the cell into shadowed and non-shadowed areas, we propose to divide the cell into circles with different radii. Shadows are then classified to these circles as shown in Figure 3.

Finally, we need to mention that performing theoretical estimation of shadowed areas according to the procedure described above there could be the case when two or more shadows overlap as shown in Figure 4, where different fillings denote doubly and triply overlapped shadowed areas. In this case RLASS in the area where shadows overlap could be different compared to RLASS in non-overlapping regions of these areas. In this case the propagation loss in regions where two or more shadows overlap needs to be computed using different pass loss exponents.

\subsection{The small-scale propagation model}

We parameterize small-scale propagation models using the histogram matching method. Depending on presence of LOS in the area $i$, process $\left\{R_{i}(n), n=0,1, \ldots\right\}$ has either Rician or Rayleigh distribution with mean $E$ $\left[R_{i}\right]=L_{i}$. Setting the number of states of the Markov chain in each state of the mobility model to one $(H=1)$ and choosing $\Delta f$ small enough we capture these distributions as

$$
F_{R, i}(k \Delta f)(\Delta f)=\frac{p_{k}}{x_{k+1}-x_{k}} .
$$

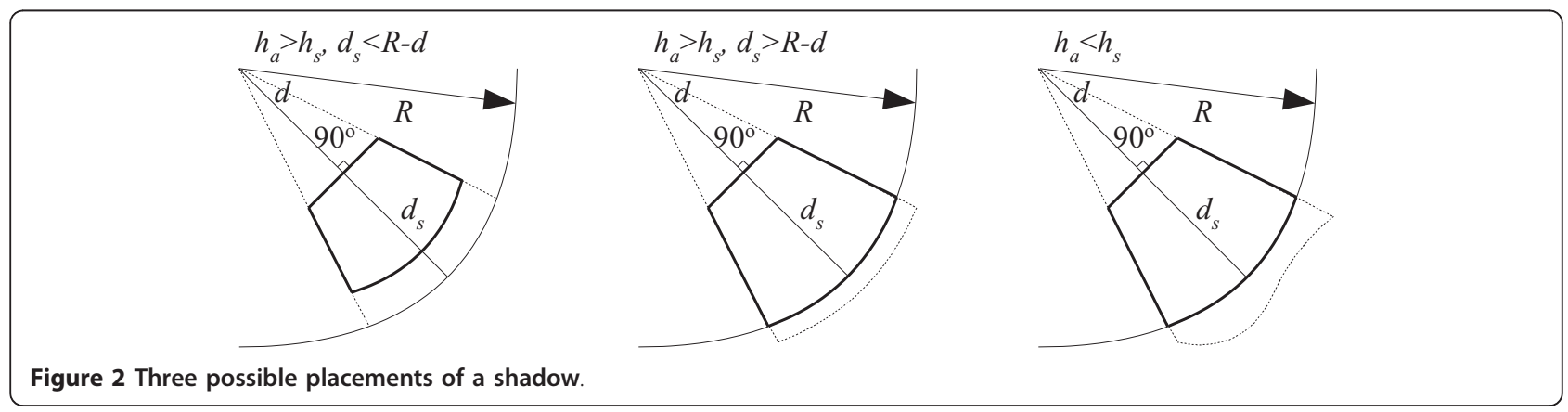


where $p_{k}=\int_{x_{k}}^{x_{k+1}} f_{X}(x) d x, f_{X}(x)$ is either Rayleigh or Rician distribution, $x^{k}$ and $x_{k+1}$ are lower and upper values for interval $k$. Alternatively, setting $H$ and solving the inverse-eigenvalue problem one can capture autocorrelation 1 and solving the inverse-eigenvalue problem one can capture autocorrelation properties of the received signal strength.

\section{Extensions and notes}

\subsection{Signal-to-interference ratio}

For a model to be useful, in addition to the received signal strength we have to take into account the level of noise and consider the signal-to-interference (SI) ratio.

The interference at the receiver is given by $I=W+U$, where $W$ the thermal noise, $U=\sum_{l=1}^{L} U(l)$ is received signal strength from $L$ interfering base stations. $W$ is usually assumed to be constant. $U$ is stochastic, depends on the distance and propagation path between the receiver and transmitters of interfering cells. Assume that $U$ is constant for any state of the mobility model. The interference process $\{U(n), n=0,1, \ldots\}, U(n)\left\{U_{1}, U_{2}\right.$. . $\left.\ldots, U_{M}\right\}$, is modulated by $\left\{S_{P}(n), n=0,1, \ldots\right\}$. We determine the mean propagation loss $E\left[L\left(m_{i j}\right)\right]$ for a given area $i$ and interfering transmitter $j$ as a function of distance them, $m_{i j}$, using (6). $E\left[L\left(m_{i}\right)\right]$ is then related to RLASS and 'mean other cells interference' is computed for areas $i$ as $U_{i}=\sum_{l=1}^{L} U_{i}(l)$.

Adding a constant $W$ to $\{U(n), n=0,1, \ldots\}$ affects the mean only allowing to consider the interference process $\{I(n), n=0,1, \ldots\}, i=1,2, \ldots, M$. The resulting signal-to-interference process is given by

$$
Y(n)= \begin{cases}\left(R_{1}-I_{1}\right)(n), & S_{P}(n)=1, \\ \cdots & \ldots \\ \left(R_{M}-I_{M}\right)(n), & S_{P}(n)=M,\end{cases}
$$

where $I_{i}=W+\sum_{l=1}^{L} U_{i}(l), i=1,2, \ldots, M$.

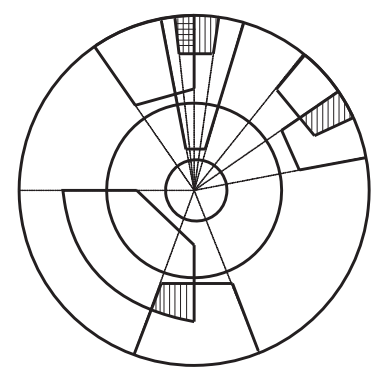

Figure 4 Doubly and triply overlapped shadowed areas.

\subsection{Attraction points}

Notice that in our model movement of the user is homogenous in time and depends on the size of areas only, see (3). However, in addition to the size of the areas other factors may affect movement of the user of the landscape. One of the most important is the socalled attraction points in a cell, i.e., places (areas in our terminology) where a user may spent significant amount of time compared to other areas. Taking into account the effect of such attraction points is equivalent to introducing non-homogeneity to the process of user movement between areas, that is, making movement to depend on some additional factors. Formally, it can be done introducing a new multipliers $g_{i}, i=1,2, \ldots, M$, each of which corresponds to a certain area into (3).

\subsection{The scope of application}

We would like to note that when micro or picocells scenarios are considered the areas we introduced in this article are almost impossible to define. The reason is that in such cells attraction points seem to play much more important role compared to the size of areas. This is clearly seen considering "office" scenario, where employees move between pre-fixed locations such as working places, meeting rooms, etc. In this case instead of parameterizing our model based on sizes of areas we need to consider attractiveness of various locations and
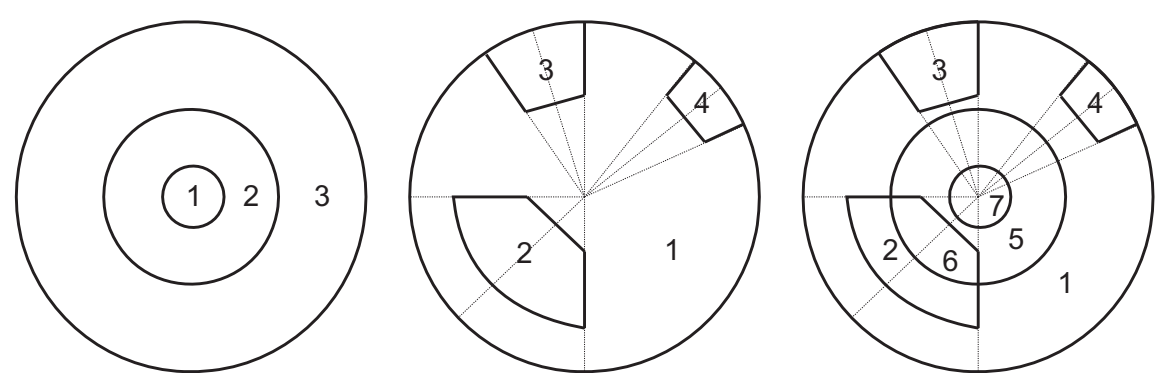

Figure 3 Example of direct estimation of shadowed areas. 
inter-location movement of users developing new expressions for estimating inter-location movements. Since there could be a number of location-specific factors involved into estimation of these probabilities we do not provide formulas for micro and picocells.

Our model was originally proposed for macrocell environments where it is possible to distinguish between different areas with quantitatively and/or qualitatively different received signal strength. The theoretical parametrization of the model is suitable for suburbs and country-side areas. When measurements of RLASS are available the model can be applied in city-center scenarios. The only difference is that in addition to sizes of areas we also need to take into account possible tunneling effects. Notice that even in this case we can use theoretical parametrization. However, instead of propagation models specified in $[1-3,10]$ we need to use special models suitable for such environment. Finally, as mentioned above with some modifications this model can be applied to microcell and picocell scenarios where a user moves between a number of attraction points. Applying this model in such scenarios additional care should be taken as even small travel distances may lead to drastic changes in small-scaled propagation characteristics due to complicated reflection and scattering observed in the buildings.

\section{Testing assumptions}

Unfortunately due to unavailability of data regarding mobility of users on the landscape we were not able to test closeness of the proposed mobility model and empirical data. Aside from this, one of the most important assumption we take in this article is that the received signal strength at a certain separation distance from the transmitter is indeed stationary while when changing the location different characteristics of the received signal strength distribution changes. To test this assumption we carried out experiments with wireless local area network (WLAN) networks operating in infrastructure DCF mode according to IEEE 802.11b standard at 11 Mbps (DSSS) in laboratory environments. The access point is installed in the corridors with rooms along both sides of it. The main points of interest were characteristics of the received signal strength process at a certain separation distance from the transmitter and its changes as users move between different attraction points. Signal strength observations were measures with $1 \mathrm{~ms}$ granularity and then averaged over $0.5 \mathrm{~ms}$ time intervals.

To test our assumption regarding stationary nature if the received signal strength two rooms were arbitrarily chosen and the signal was measured for a long duration of time. Two samples gather as a result of these experiments are shown in Figure 5. Just observing these traces no definitive conclusions regarding stationarity of the SNR process at a certain separation distance from the transmitter can be made. For this reason we performed detailed statistical analysis as discussed below.

Histograms of relative frequencies service as estimates of empirical distributions and their approximations by Normal distribution are show in Figure 6. As one may observe approximations are quite good. Testing the null hypothesis about Normal distribution of data using $\chi^{2}$ test confirmed it with the level of significance, $\alpha$, set to 0.1 . Notice that for the second trace the null hypothesis is accepted even when $\alpha=0.05$. Tests performed for other rooms (not shown here due to space constraints) also confirm this approximation. We would like to note that one should not be surprised with normality of observations. Recall that we averaged the received signal strength over $0.5 \mathrm{~s}$. intervals providing sufficient aggregation level for central limit theorem to hold.

In addition to distributional properties discussed above the process is found to be autocorrelated as highlighted as normalized autocorrelation functions (NACF) shown in Figure 6c, d. The Ljung-Box test (a special type of portmanteau test) performed with the level of significance $\alpha=0.1$ showed that first three lags of the first sample and the first lag of the second one are statistically different from zero with level of confidence $\alpha$ $=0.1$. Observing the figures we see that the both functions decay geometrically fast.

Note that the above mentioned analysis does not allow us to conclude that the process at a certain separation distance is covariance stationary. Unfortunately, there are no well-behaved tests to access stationarity of stochastic processes. We use the following two-steps procedure. First of all, observe that explicitly assuming covariance stationarity the above mentioned analysis allows to model SNR data at a certain separation distance from the transmitter using autoregressive process of order $1, \operatorname{AR}(1)$, in the form $Y_{i}=\varphi_{0}+\varphi_{1} Y_{i-1}+\varepsilon_{i}, i=$ $0,1, \ldots$, where $\varphi_{0}$ and $\varphi_{1}$ are some constants, $\varepsilon_{i}$ are independently and identically distributed random variables having the same normal distribution with zero mean and variance $\sigma^{2}[\varepsilon]$. Thus, we used aposteriori change-point exponential weighted moving average test for $\operatorname{AR}(1)$ (see $[11,12]$ ) to detect change in the mean of SNR data. For our observations no changes have been observed in both samples. However, this does not necessarily imply that there are no changes in other parameters of the process. To address this situation we divided each sample into three different subsamples and compare their distributional and correlational properties. It appeared that using $\chi^{2}$ test with the level of significance set to $\alpha=0.1$ all the subsamples drawn from a certain sample are homogenous, i.e., have the same 1D distributions. Usage of rather big value of $\alpha$ is explained 


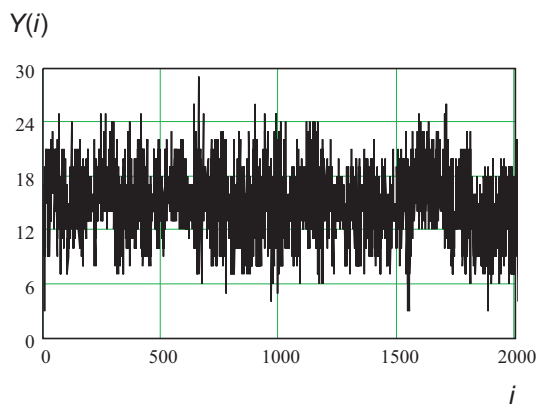

(a) Sample 1
$Y(i)$

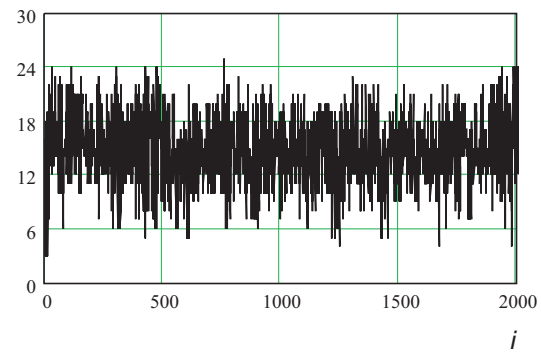

(b) Sample 2

Figure 5 Samples of SNR observations (location is fixed).

by presence of correlation in subsamples. To conceal the effect of correlation we also used every second observation in each subsample to compute empirical distributions. The turning point test demonstrated that the hypothesis about white noise should be rejected with the level of significant $\alpha=0.05$ indicating that there is memory in each of those sample. Finally, in each subsample NACF decayed geometrically fast. The reason for performing the turning point test instead of, e.g., Ljung-Box portmanteau test is that it is unreasonable to expect the same number of lags having statistically significant correlation in samples of small sizes. Although these observations do not strictly prove that SNR process at a certain separation distance from the transmitter is covariance stationary they provide enough evidence that first and second-order characteristics likely remain unchanged.

Recall that in addition to covariance stationarity of SNR process as a certain separation distance from the

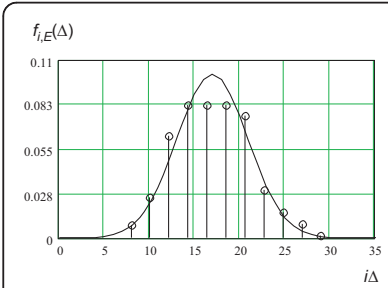

(a) Sample 1

$K_{Y}(i)$

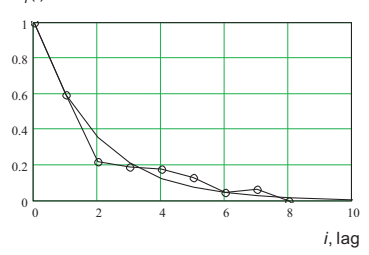

(c) Sample 1

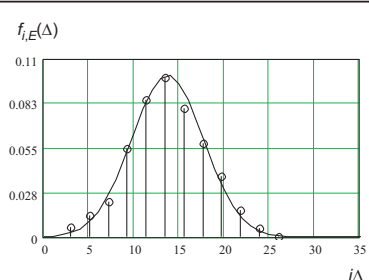

(b) Sample 2

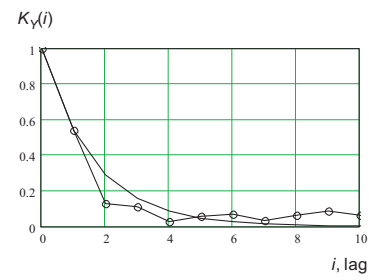

(d) Sample 2
Figure 6 Histograms and NACFs of SNR observations and their approximations. transmitter we also assumed that the mean (and possibly distribution) changes as user moves between different locations. To back up our assumption we performed the following experiments. A mobile station was in stationary position for some noticeable amount of time. Then, a user moved into another office room and the station again remained in stationary state for some amount of time. Two arbitrarily chosen SNR samples obtained by changing the location of the user during the measuring process are shown in Figure 7. Observing these traces one may notice that the change in the received signal strength happens almost instantaneously. The reason is that the distances between attraction points in office environment are rather short (recall that the granularity of SNR measurements is $0.5 \mathrm{~s}$ ).

Results of EWMA change point test are shown in Figure 8 for two values of smoothing parameters $\gamma$. In all demonstrated figures the change in SNR statistics is detected. Moreover, our results show that changes are detected for all values of smoothing parameters $\gamma$ that are less than 0.1 implying that there is no need to access accuracy of the test using average run length (ARL) statistics. Note that change-point statistical tests are rather coarse in general. To ensure that the change in some parameters of the distribution happened we compared distributional characteristics of two subsamples drawn from each sample. To get these subsamples we used results of EWMA test to exclude those observations occurring at the change point. $\chi^{2}$ test performed with the level of significance set to $\alpha=0.05$ showed that the null hypothesis about the same empirical distribution should be rejected.

Note that the above mentioned statistical analysis demonstrate that (i) SNR process at a certain separation distance from the transmitter is close to covariance stationary (ii) SNR process may quantitatively change when user changes its location. Supplementing the above mentioned analysis with mobility model between attraction points we a particular case of the mobility- 


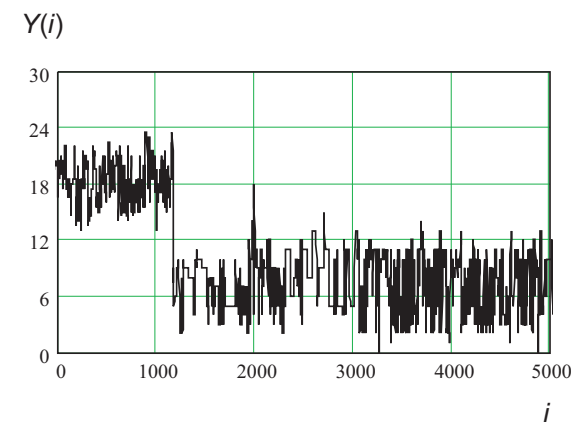

(a) Sample 1

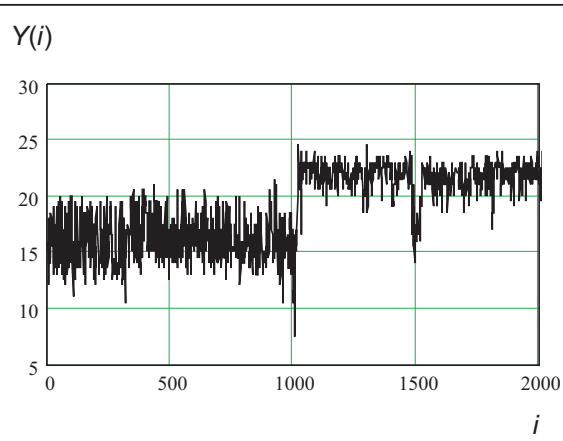

(b) Sample 2

Figure 7 Time series of SNR observations (location is changed).

dependent propagation model introduced in this article. Although these test have been performed in office environment using IEEE 802.11b WLAN we can expect the same statistical behavior for public wireless networks. Still there are important differences. First of all, due to larger spaces covered by these networks in addition to attraction points we need to consider areas as described in Section 2. Secondly, changes in characteristics of the received signal strength are not expected to happen drastically as user moved between areas and/or attraction points.

\section{Conclusions}

We developed an extension for wireless channel modeling techniques explicitly taking into account the movement of the user between areas with different smallscale propagation characteristics. Although a single cell environment has been considered, results can be extended to multiple cells scenario.

The model is suitable for performance evaluation of applications in presence of signal strength changes caused by movement of the user. Although the Markov structure of the model allows for analytical performance

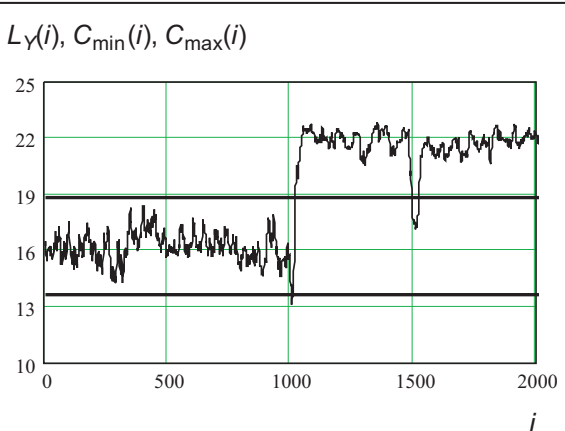

(a) Sample 1: $\gamma=0.1$

$L_{Y}(i), C_{\min }(i), C_{\max }(i)$

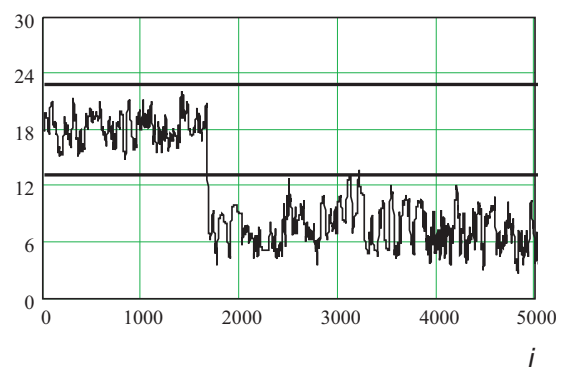

(c) Sample 2: $\gamma=0.1$
$L_{Y}(i), C_{\min }(i), C_{\max }(i)$

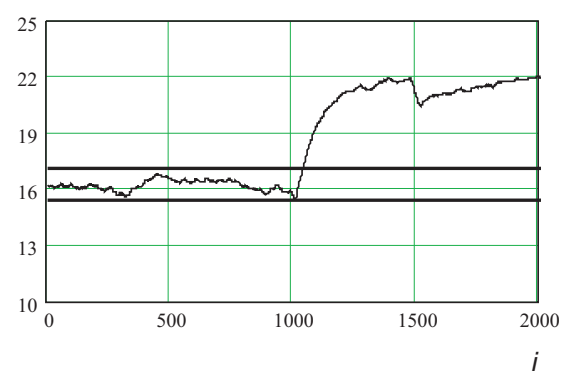

(b) Sample 1: $\gamma=0.01$

$L_{Y}(i), C_{\min }(i), C_{\max }(i)$

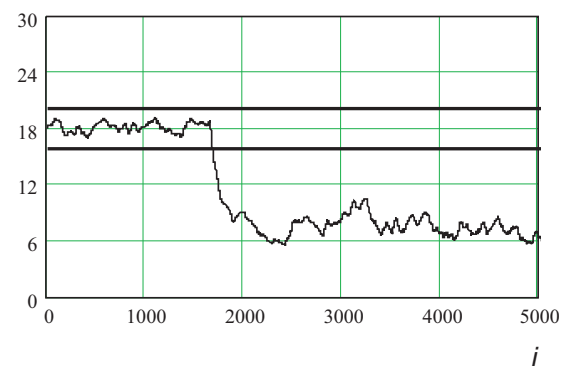

(d) Sample 2: $\gamma=0.01$

Figure 8 Time series of SNR observations (location is changed). 
modeling, large state space may restrict its usage to simulation studies. We note that the model can be extended to capture propagation-dependent behavior. For example, the current modulation and coding scheme as a function of mobility of the user can be modeled.

\section{Competing interests}

The authors declare that they have no competing interests.

Received: 24 February 2011 Accepted: 2 April 2012

Published: 2 April 2012

\section{References}

1. T Okumura, E Omori, K Fakuda, Field strength and its variability in VHF and UHF land mobile service. Rev Electr Commun Lab. 16(9/10):825-873 (1968)

2. M Hata, Empirical formula for propagation loss in land mobile radio services. IEEE Trans Veh Tech. VT-29(3):317-325 (1980)

3. M Feuerestein, K Blackard, T Rappaport, S Seidel, H Xia, Path loss, delay spread, and outage models as functions of antena height for microcellular system design. IEEE Trans Veh Tech. 43(3):487-498 (1994). doi:10.1109/ 25.312809

4. A Saleh, R Valenzuela, A statistical model for indoor multipath propagation. IEEE JSAC. 5(2):128-137 (1987)

5. T Rappaport, Statistical channel impulse response models for factory and open plan building radio communication system design. IEEE Trans Commun. 39(5):794-806 (1991). doi:10.1109/26.87142

6. D Durgin, T Rappaport, Theory of multipath shape factors for small-scale fading wireless channels. IEEE Trans Ant Propag. 48, 682-693 (2000). doi:10.1109/8.855486

7. T Rappaport, Wireless Communications: Principles and Practice, 2nd edn (Prentice Hall, Upper Saddle River, 2002)

8. $\quad$ P Green, R Sibson, Computing Dirichlet tesselations in the plane. Comput J. 21, 168-173 (1978)

9. S Seidel, Path loss, scattering and multipath delay statistics in four european cities of digital cellular and microcellular radiotelephone. IEEE Trans Veh Tech. 40(4):721-730 (1991). doi:10.1109/25.108383

10. Digital mobile radio, towards future generation systems. COST 231 Final Report, EUR18957. (1999)

11. D Moltchanov, State description of wireless channels using change-point statistical tests. in Proc WWIC'2006, vol. 3970. (Bern, Switzerland, 2006), pp. 275-286

12. W Schmid, A Schone, Some properties of the EWMA control chart in the presence of auto-correlation. Ann Stat. 25(3):1277-1283 (1997). doi:10.1214/ aos $/ 1069362748$

doi:10.1186/1687-1499-2012-130

Cite this article as: Moltchanov: Mobility-dependent small-scale

propagation model for applied simulation studies. EURASIP Journal on Wireless Communications and Networking 2012 2012:130.

\section{Submit your manuscript to a SpringerOpen ${ }^{\mathcal{O}}$ journal and benefit from:}

- Convenient online submission

- Rigorous peer review

- Immediate publication on acceptance

- Open access: articles freely available online

- High visibility within the field

- Retaining the copyright to your article

Submit your next manuscript at $>$ springeropen.com 